\title{
La ruralité à l'épreuve du navettage : La recomposition de l'espace au Saguenay-Lac-St-Jean
}

\author{
Martin Simard $^{1}$, Yves Chiricota ${ }^{2}$ et Carl Brisson ${ }^{3}$ \\ Université du Québec à Chicoutimi \\ François Queyroi ${ }^{4}$ \\ Université de Bordeaux I et II
}

\section{INTRODUCTION}

\section{Que l'on parle de mégalopole, de métapole ou de métropolisation, cette évolution remet en cause la nature fondamentale des territoires et les classifications territoriales (urbain, rural, banlieue, etc.).}

Les métropoles tendent à se fragmenter et à s'étaler en insérant dans leur aire d'influence de vastes territoires suburbains et ruraux. Au-delà des traditionnelles relations de polarisation commerciale ou de services publics entre les villes et les campagnes, le navettage, ou les flux quotidiens de déplacements résidence-travail, posent les questions de l'organisation du territoire du quotidien et des frontières de l'agglomération. Que l'on parle de mégalopole, de métapole ou de métropolisation, cette évolution remet en cause la nature fondamentale des territoires et les classifications territoriales (urbain, rural, banlieue, etc.). Au cours des années 1960, la motorisation des rapports aux territoires dans les sociétés modernes a déjà été perçue comme destructrice de communautés, en particulier en Amérique du Nord. Sommes-nous maintenant rendus à l'étape de la destruction / recomposition de l'urbanité et, surtout, de la ruralité ? Il est vrai que ces immenses bassins d'emplois que sont devenus les territoires métropolitains et régionaux tendent à devenir des espaces politiques semi-autonomes à travers les regroupements municipaux, la création de structures politiques régionales et le rééchelonnement des pouvoirs au sein des instances publiques. Toutefois, la diversité des paysages et des modes d'occupation du sol ainsi que la nomenclature que les populations se sont appro- priées (quartier, village, ville, etc.) cachent une recomposition profonde des espaces régionaux.

L'augmentation des déplacements et la complexification des réseaux d'échanges témoignent des changements en cours dans divers types de territoires. Néanmoins, on peut y observer des régularités ${ }^{5}$. Nos travaux consistent donc à étudier les processus et les dynamiques de recomposition au sein des villes et des territoires périphériques à travers le navettage. Il ne s'agit pas d'analyser les liens entre le navettage et les caractéristiques individuelles des ménages, mais plutôt les liens aux territoires. Par ailleurs, nous ne visons pas à comprendre les liens entre la forme urbaine ou métropolitaine car les espaces régionaux sont polycentriques et «dispersés », par nature. Plus précisément, notre objectif est de mesurer l'intensité, les formes et l'évolution du navettage à l'intérieur de territoires périphériques dotés d'une ville moyenne. De manière spécifique, nous tentons de schématiser les flux de navettage au Saguenay-Lac-Saint-Jean.

\section{Les transformations liées à la motorisation et au navettage touchent également les villes petites et moyennes, et les territoires ruraux avoisinants, même si les conditions de la métropolisation n'y sont pas toutes réunies.}

À l'instar d'autres chercheurs ${ }^{6}$, nous considérons que les transformations liées à la motorisation et au navettage touchent également les villes petites et moyennes et les territoires ruraux avoisinants, même si les conditions de la métropolisation n'y sont pas toutes réunies. À titre d'exemple, la 
région métropolitaine de recensement (RMR) de Saguenay au Québec regroupe plus de 150000 habitants et celle-ci polarise un vaste Hinterland s'étendant sur l'ensemble de la région du Saguenay-Lac-Saint-Jean, et même au-delà. Cependant, Saguenay se localise en milieu périphérique, c'est-à-dire à l'extérieur de la vallée du Saint-Laurent, le berceau historique du Québec et du Canada au peuplement plus dense. Certains facteurs semblent défavoriser les villes moyennes périphériques et participer à leur stagnation, voire à leur déclassement dans l'espace-Monde contemporain ${ }^{7}$. Ces difficultés sont liées à leur positionnement relatif, à une base économique axée principalement sur les ressources naturelles et à leur faible habileté à attirer des immigrants internationaux.

\section{Le Saguenay-Lac-Saint-Jean semble être un laboratoire pertinent pour l'observation du navettage à l'échelle régionale. Ce territoire forme une enclave naturelle séparée des autres sections de l'écoumène québécois par des espaces forestiers.}

Malgré son contexte périphérique et la décroissance relative de sa ville principale, le Saguenay-Lac-Saint-Jean semble être un laboratoire pertinent pour l'observation du navettage à l'échelle régionale. Ce territoire forme une enclave naturelle séparée des autres sections de l'écoumène québécois par des espaces forestiers, ce qui permet de réduire les effets de frontières dans notre regard sur la mobilité quotidienne. En plus de Saguenay, cet espace régional comporte quatre petits pôles urbains qui nous autorisent à enrichir l'analyse et à interpréter les effets de taille et de distance sur le navettage. En termes de cheminement des idées, nous traiterons d'abord brièvement des concepts d'urbain et de rural qui apparaissent plutôt obsolètes à l'ère de la mondialisation. En deuxième lieu, nous discuterons des aspects méthodologiques de la recherche. Troisièmement, nous décrirons les flux, les patrons et l'évolution du navettage sur le territoire cible à l'aide de cartes et de tableaux. Finalement, nous tenterons de catégoriser les milieux ruraux de la région à l'étude à travers une typologie intégrant plusieurs variables, dont le navettage.

\section{LA DICHOTOMIE URBAIN-RURAL À L'ÉPREUVE DU NAVETTAGE}

Bien qu'ils soient profondément ancrés dans l'imaginaire collectif, les concepts d'urbain, de rural, de ville ou de campagne semblent plutôt fragiles lorsque soumis à l'analyse, en particulier en regard de la réalité socioéconomique et culturelle des sociétés modernes avancées. Dans le passé, la ville se distinguait assez aisément du campement ou du village par sa taille, sa forme et ses fonctions économiques et politiques. Il n'en est plus de même aujourd'hui alors que les établissements ruraux grossissent, se transforment qualitativement et adoptent des formes architecturales similaires à celles des milieux urbains. De plus, les limites de la ville deviennent ardues à déterminer avec le développement des banlieues et l'urbanisation de la proche campagne. Aux États-Unis, la majorité de la population urbaine vit maintenant en milieu suburbain ${ }^{8}$ et ce phénomène prend des proportions semblables au Canada. Cette banlieue est de plus en plus lointaine et elle correspond souvent à la recolonisation de noyaux villageois antérieurs.

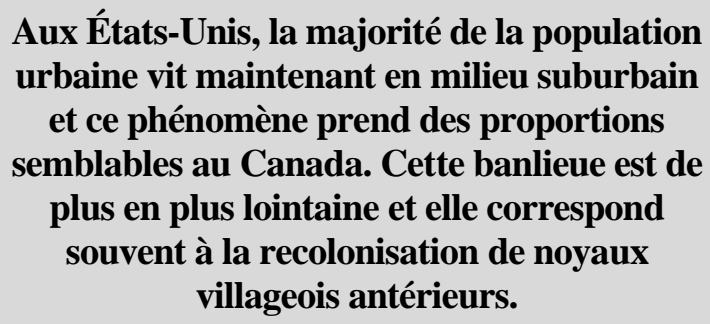

En fait, il est de plus en plus difficile de situer clairement la différence entre l'urbain et le rural. Pour des fins de classification, de compilation statistique ou de mise en œuvre de politiques publiques, les critères suivants sont généralement utilisés : la taille ou un seuil de population, la densité de la population et des bâtiments ainsi que la diversité des activités économiques. Malgré ses aspects réducteurs, le critère de la taille de la population demeure le plus simple et le plus utilisé. Par exemple, pour Statistique Canada, "Une région urbaine est un territoire [municipal] d'au moins 1000 habitants 
ayant une densité de population d'au moins 400 habitants au kilomètre carré, d'après les chiffres de population du recensement le plus récent ». Il s'agit d'une convention et non d'une définition de nature scientifique. Par ailleurs, le même organisme public a développé d'autres concepts (région métropolitaine de recensement ou RMR, zone d'influence métropolitaine ou ZIM, etc.) pour rendre compte de l'évolution des formes d'occupation des territoires.

L'urbanisation contemporaine prend la forme dispersée dans le contexte d'une société mobile. Cette mobilité est associée au phénomène de métropolisation dans les grands pôles urbains, mais le débat reste ouvert sur la pertinence ou non de l'utilisation de ce terme pour les villes de tailles intermédiaires. Pendant spatial de la mondialisation, la métropolisation peut être définie comme étant « un processus de sélection des territoires, à travers la concentration des hommes et des activités dans les villes les plus importantes et les plus dynamiques ${ }^{9}$. Cette sélection opèrerait également à l'échelle intra-urbaine. Ainsi, le processus de métropolisation conduirait «à la formation d'espaces urbains de plus en plus fragmentés remettant en cause les modèles de répartition des citadins ou des activités par rapport à un centre unique ${ }^{10}$. À partir de l'exemple de Nice, Fusco et Scarella ${ }^{11}$ catégorisent en deux les mécanismes spatiaux de la métropolisation à l'échelle régionale : premièrement, la périurbanisation des espaces agricoles et naturels par les résidences principales; deuxièmement, la colonisation liée aux loisirs de l'arrière-pays par les résidences secondaires. En conséquence, les seuls milieux « ruraux » qui ne sont pas affectés par la déprise sont alors les territoires périurbains ou les espaces de villégiature.

L'écoumène des États postindustriels se complexifie donc, alternant sans ordre apparent les anciens foyers d'urbanisation plus denses, les zones suburbaines de diverses générations, les enclaves ou friches industrielles, les espaces ruraux

\section{MÉTHODOLOGIE}

L'indicateur utilisé pour témoigner de la recomposition des territoires locaux au sein d'une aire polarisée par une ville moyenne est le navettage ou les flux quotidiens de travailleurs soumis à la villégiature ou les aires dévitalisées. Ce phénomène est particulièrement visible dans les régions en forte croissance comme le sud de la Californie, où l'école de Los Angeles a remis en cause les modèles de la structure urbaine ${ }^{12}$. Cette complexité des formes spatiales et l'expansion géographique d'aires d'influences urbaines, qui se télescopent et forment de vastes bassins d'emplois, en viennent à questionner les concepts mêmes d'urbain et de rural. Pourtant, les expressions « hinterland» ou «champ urbain » illustrent depuis longtemps la polarisation des villes sur les espaces limitrophes, un rayonnement qui peut atteindre des dizaines voire des centaines de kilomètres de distance. Néanmoins, ce traditionnel rayonnement commercial et administratif sur de larges périmètres intègre maintenant les flux quotidiens de navettage.

Les dynamiques spatiales à l'ère de la métropolisation ont été étudiées par l'intermédiaire des fonctions supérieures des centres d'affaires, de l'emploi et du rayonnement commercial ou de la construction résidentielle. Nous nous concentrerons sur le phénomène de navettage à l'instar d'autres chercheurs ${ }^{13}$. Au Canada, le navettage est le fondement des notions de régions métropolitaines de recensement (RMR) et d'agglomérations de recensement (AR). Ces unités géostatistiques témoignent de la caducité des frontières administratives locales par rapport aux processus socioéconomiques. De plus, la nouvelle classification des localités en zones d'influence métropolitaine (ZIM) par Statistique Canada illustre la montée en échelle des dynamiques d'agglomération. Sans traiter directement du débat sur la métropolisation en contexte de villes moyennes, notre analyse souhaite aller au-delà du concept de ZIM en mesurant, plus précisément, les flux de navettage, leur évolution dans le temps de même que leur relation avec diverses variables socioéconomiques qui façonnent la diversité géographique des territoires régionaux.

vers une municipalité autre que celle de résidence. Celui-ci nous ramène au cœur même des bouleversements sociospatiaux de notre époque; «signifiant davantage qu'un simple phénomène 
de croissance démographique des grandes agglomérations, la métropolisation se conjugue à l'étalement urbain, à la fragmentation des espaces fonctionnels et à la recomposition des milieux de vie, mettant l'accent sur une mobilité accrue des individus, de l'information et des activités $»^{14}$. La croissance du navettage apparaît alors comme un élément majeur de l'urbanisation contemporaine, à la fois en tant que reflet des réaménagements économiques et fonctionnels des espaces urbains et régionaux ainsi qu'à titre d'illustration des changements sociodémographiques et d'une ouverture culturelle de plus en plus forte à la mobilité quotidienne.

Nous comptons étudier le navettage ou les flux quotidiens de travailleurs entre Saguenay et les autres municipalités localisées dans la région administrative du Saguenay-Lac-Saint-Jean (voir figure 1). Nos travaux s'inscrivent dans la continuité des recherches déjà réalisées sur ces mêmes thèmes et territoires ${ }^{15}$. Dans une perspective pratique, les données utilisées proviennent de compilations spéciales de Statistique Canada. Celles-ci sont issues des recensements de population de 1996 et de 2006. Les données concernent les 50 localités de la région administrative du Saguenay-Lac-Saint-Jean, soit 49 municipalités locales et une réserve autochtone voisine de la ville de Roberval (Mashteuiatsh). Nos analyses focaliseront sur les municipalités de plus de 10000 habitants qui sont considérées urbaines aux fins de cette étude, soit principalement Saguenay mais aussi Alma, DolbeauMistassini, Saint-Félicien et Roberval. Saguenay a le statut de région métropolitaine de recensement (RMR), Alma et Dolbeau-Mistassini celui d'agglomération de recensement (AR) alors que les deux autres villes ne sont pas reconnues dans les classements de Statistique Canada malgré leurs populations respectives dépassent quelque peu 10000 habitants.

\section{Carte 1 - Localisation et population des 50 localités du Saguenay-Lac-St-Jean}

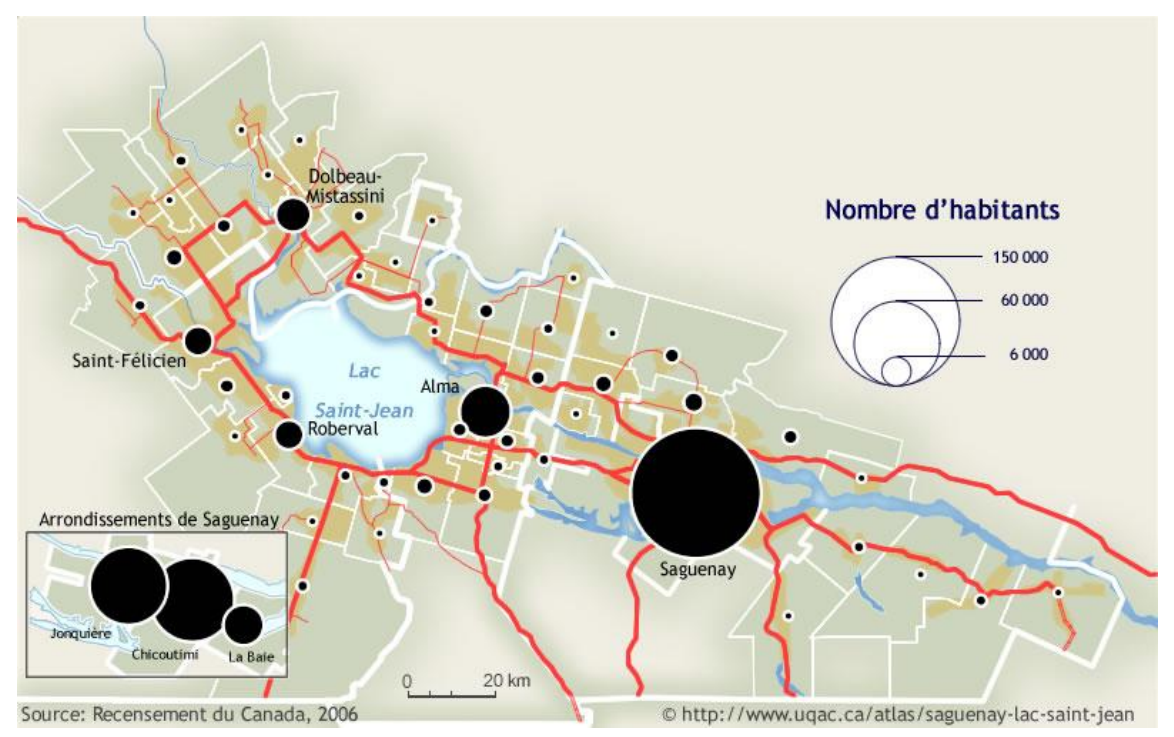

En ce qui concerne la démarche scientifique, les données sur le navettage ont d'abord fait l'objet de traitements statistiques. Par la suite, ils ont été regroupés en classes en fonction de seuils standardisés dans les distributions pour les deux années afin de représenter et de décrire leurs patrons géographiques et leur évolution historique. Nous avons aussi effectué des corrélations entre l'intensité et l'évolution des flux de navettage, d'une part, et certains variables telles que la population active travaillant dans le secteur tertiaire dans les municipalités émettrices, d'autre part. L'indice de développement du ministère des Affaires municipales, des régions et de l'occupation du territoire $\left(\right.$ MAMROT) ${ }^{16}$ a également été utilisé pour les croisements de données. Finalement, des données sur la construction résidentielle ont été mobilisées pour une étude plus approfondie des dynamiques territoriales. 
Cela nous a amené à réaliser une typologie des municipalités rurales du Saguenay-Lac-SaintJean à partir des divers indicateurs disponibles. Pour créer la typologie, nous avons utilisé une méthode de classification ascendante hiérarchique en considérant le navettage, la distance euclidienne, l'indice de dévitalisation du MAMROT et la construction résidentielle des dernières années. À chaque itération, deux municipalités (ou deux groupes de municipalités) sont agrégés de manière à minimiser la perte d'information ou d'inertie dans l'échantillon en utilisant le critère de Ward. À partir de cette hiérarchie, nous avons décidé de sélectionner quatre groupes de municipalités rurales. Ce choix est justifié par la volonté de ne pas obtenir des groupes de trop petite taille (par rapport à la taille de l'échantillon). De plus, la perte d'information est faible avec ce découpage : le critère de qualité du $\mathrm{R}^{\wedge} 2$ ajusté vaut dans ce cadre 0,89 . Néanmoins, le nombre d'unités territoriales, soit 45 , est très faible d'un point de vue statistique. Ce constat doit venir relativiser les conclusions que nous tirerons des corrélations et de la classification typologique.

\section{LE NAVETTAGE AU SAGUENAY-LAC-ST-JEAN}

\section{Le potentiel d'attraction des villes est directement proportionnel à leurs tailles. À elle seule, Saguenay s'accapare de 5,49\%$$
\text { de la population active occupée, soit }
$$$$
\mathbf{2 8 , 4 8} \% \text { de tous les navetteurs et } \mathbf{4 2 , 4 5} \%
$$ des navetteurs vers les villes.}

L'analyse des données de navettage fait ressortir diverses tendances générales. Premièrement, notons que la population active occupée de cette région administrative québécoise - 108540 personnes ou 39,6\% de la population totale en 2006 - se déplace quotidiennement à l'extérieur de sa municipalité de résidence pour le travail dans une proportion de 19,28\% (voir le tableau 1). Le navettage est particulièrement fort en milieu rural $^{17}$ avec un taux de $61,65 \%$. Ce phénomène s'accroît dans le temps avec une augmentation de 3,97\% entre 1996 et 2006. Malgré tout, le taux de rétention des travailleurs par municipalité locale demeure élevé à plus de $80 \%$, en particulier à l'intérieur des espaces urbains. Deuxièmement, les flux de navettage expriment la prédominance claire de Saguenay et des quatre autres pôles urbains de la région administrative en tant que points d'attraction (voir la carte 2). Ceux-ci attirent $12,94 \%$ de la population active occupée ou près du $2 / 3$ des navetteurs. D'ailleurs, le potentiel d'attraction des villes est directement proportionnel à leurs tailles. À elle seule, Saguenay s'accapare de $5,49 \%$ de la population active occupée, soit $28,48 \%$ de tous les navetteurs et $42,45 \%$ des navetteurs vers les villes.

Tableau 1 - Taux de navettage et de rétention par territoires au Saguenay-Lac-St-Jean (1996-06)

\begin{tabular}{|l|c|c|c|c|c|c|}
\hline $\begin{array}{l}\text { Population active } \\
\text { occupée }\end{array}$ & $\begin{array}{c}\text { Taux de } \\
\text { navettage } \\
\mathbf{2 0 0 6}\end{array}$ & $\begin{array}{c}\text { Taux de } \\
\text { navettage } \\
\mathbf{1 9 9 6}\end{array}$ & $\begin{array}{c}\text { Différence } \\
\text { de \% 1996- } \\
\mathbf{2 0 0 6}\end{array}$ & $\begin{array}{c}\text { Taux de } \\
\text { rétention } \\
\mathbf{2 0 0 6}\end{array}$ & $\begin{array}{c}\text { Taux de } \\
\text { rétention } \\
\mathbf{1 9 9 6}\end{array}$ & $\begin{array}{c}\text { Différence } \\
\text { de \% 1996- } \\
\mathbf{2 0 0 6}\end{array}$ \\
\hline Région du Saguenay & 10,65 & 8,32 & 2,33 & 89,31 & 91,68 & $-2,36$ \\
\hline Région du Lac St-Jean & 33,10 & 27,24 & 5,86 & 66,73 & 72,92 & $-6,18$ \\
\hline Zones rurales & 61,65 & 56,14 & 5,51 & 37,83 & 44,19 & $-6,36$ \\
\hline Zones urbaines & 8,43 & 6,55 & 1,88 & 91,60 & 93,45 & $-1,85$ \\
\hline TOTAL SLSJ & 19,28 & 15,32 & 3,97 & 80,63 & 84,74 & $-4,11$ \\
\hline
\end{tabular}




\section{Carte 2 - Évolution des flux de navettage au Saguenay-Lac-St-Jean (1996-2006)}

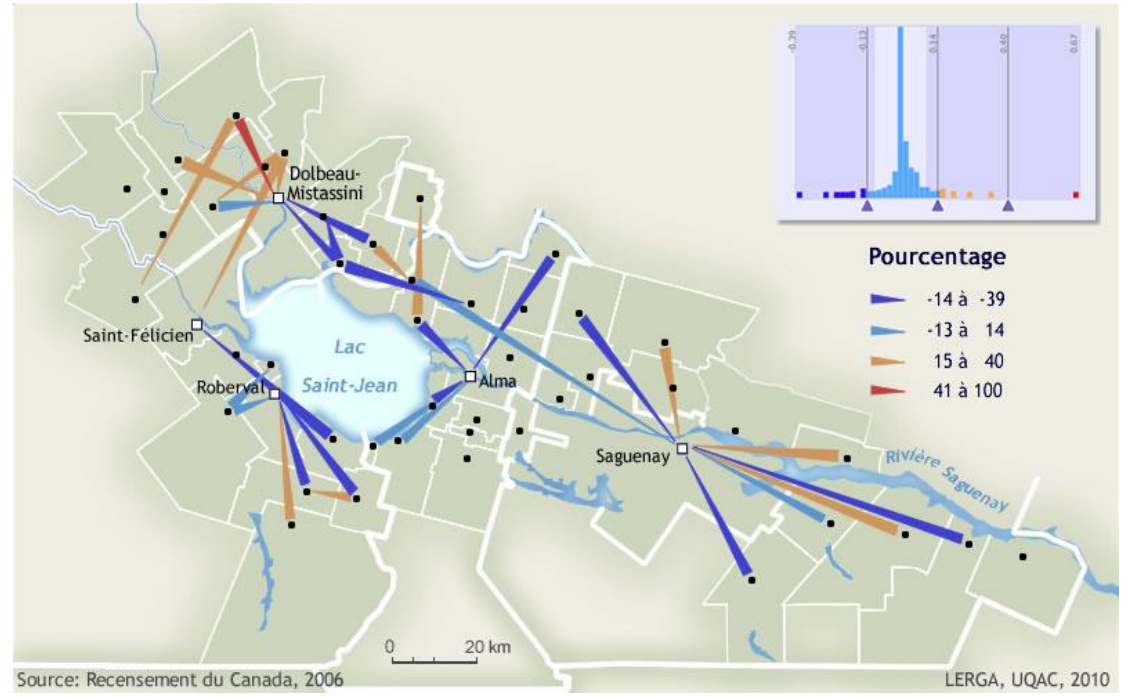

De manière plus détaillée, les municipalités de la sous-région du Saguenay sont polarisées fortement par la Ville de Saguenay avec neuf municipalités sur 14 comptant plus de $50 \%$ de navetteurs (voir le tableau 2). Entre 1996 et 2006, le taux de navetteurs à partir de ces territoires est passé de $54,34 \%$ à $59,16 \%$, soit une augmentation de près de $5 \%$. Cependant, certaines municipalités comme 1'Anse-SaintJean, Ferland-et-Boilleau de même que SaintFulgence ont vu le nombre de navetteurs diminué entre ces deux années. Cela pourrait s'expliquer par la crise dans le secteur forestier, en particulier par la fermeture de l'importante usine de pâtes et papiers de Port-Alfred dans l'arrondissement de La Baie à Saguenay (680 emplois manufacturiers). Le territoire du LacSaint-Jean voit aussi les navetteurs vers Saguenay augmenter avec un taux qui passe de $3,3 \%$ à $4,3 \%$. En outre, le secteur Est de la sousrégion du Lac-Saint-Jean est soumis à l'influence saguenéenne avec des taux de déplacements quotidiens de la population active occupée vers Saguenay qui dépassent fréquemment les $10 \%$ malgré le rayonnement prépondérant d'Alma sur ces localités qui l'avoisinent.

Tableau 2 - Flux de navettage par municipalité au Saguenay (1996-2006)

\begin{tabular}{|l|c|c|c|}
\hline $\begin{array}{l}\text { Population active } \\
\text { occupée }\end{array}$ & $\begin{array}{c}\text { \% se déplaçant à } \\
\text { Saguenay 1996 }\end{array}$ & $\begin{array}{c}\text { \% se déplaçant à } \\
\text { Saguenay 2006 }\end{array}$ & $\begin{array}{c}\text { Différence de \% } \\
\mathbf{1 9 9 6 - 2 0 0 6}\end{array}$ \\
\hline Saint-Félix-d'Otis & 67,7 & 86,5 & 18,7 \\
\hline Saint-Honoré & 70,8 & 70,3 & $-0,5$ \\
\hline Saint-Fulgence & 72,0 & 66,5 & $-5,6$ \\
\hline Rivière-Éternité & 44,8 & 65,5 & 20,7 \\
\hline Saint-David-de-Falardeau & 44,2 & 63,6 & 19,4 \\
\hline Sainte-Rose-du-Nord & 37,0 & 63,2 & 26,1 \\
\hline Ferland-et-Boilleau & 70,0 & 60,7 & $-9,3$ \\
\hline Saint-Charles-de-Bourget & 62,9 & 59,0 & $-3,9$ \\
\hline Larouche & 47,4 & 53,8 & 6,4 \\
\hline Saint-Ambroise & 45,2 & 48,4 & 3,2 \\
\hline Bégin & 34,9 & 31,4 & $-3,5$ \\
\hline Petit-Saguenay & 18,9 & 28,1 & 9,2 \\
\hline L'Anse-Saint-Jean & 35,3 & 18,8 & $-16,5$ \\
\hline TOTAL Région du Saguenay & $\mathbf{5 4 , 3 4}$ & $\mathbf{5 9 , 1 6}$ & $\mathbf{4 , 8 2}$ \\
\hline
\end{tabular}


Troisièmement, les échanges entre les pôles urbains eux-mêmes sont intenses et ils s'accroissent aussi avec le temps. On remarque des échanges importants entre Saguenay et Alma (voir le tableau 3), d'une part $(7,71 \%$ des Almatois travaillent à Saguenay en 2006) ainsi qu'entre les trois centres du nord-ouest du LacSaint-Jean, soit Dolbeau-Mistassini, SaintFélicien et Roberval, d'autre part $(8,83 \%$ des Félicinois travaillent à Roberval et alors 6,46\% font le trajet inverse sur une base quotidienne). Ces flux de déplacements quotidiens d'individus faisant partie de la population active occupée ont tendance à augmenter. Ce phénomène de compé- tition interurbaine est d'autant plus fort que les pôles sont rapprochés et de taille comparable, comme c'est le cas dans la sous-région du LacSaint-Jean. Les liens entre Saguenay et Alma se sont aussi grandement fortifiés. Il faut dire que l'érection d'une nouvelle aluminerie à Alma en 1998 et la construction de l'autoroute 70 qui relie les deux villes depuis 2003 sont deux facteurs explicatifs probants. Dans la même veine, la fermeture en 2010 de la papeterie de Dolbeau-Mistassini viendra sûrement changer la donne, dans la section nord-ouest du Lac-SaintJean, en diminuant l'attractivité de cette ville et en modifiant son profil socioéconomique.

Tableau 3 - Flux de navettage entre les cinq pôles urbains du Saguenay-Lac-St-Jean (1996-06)

\begin{tabular}{|l|c|c|c|c|c|c|c|c|c|c|}
\hline $\begin{array}{c}\text { Population } \\
\text { active } \\
\text { occupée }\end{array}$ & \multicolumn{2}{|c|}{$\begin{array}{c}\text { Navettage urbain } \\
\text { vers Saguenay }\end{array}$} & \multicolumn{2}{c|}{$\begin{array}{c}\text { Navettage urbain } \\
\text { vers Alma }\end{array}$} & \multicolumn{2}{c|}{$\begin{array}{c}\text { Navettage urbain } \\
\text { vers Dolbeau-Mist. }\end{array}$} & \multicolumn{2}{|c|}{$\begin{array}{c}\text { Navettage urbain } \\
\text { vers Roberval }\end{array}$} & \multicolumn{2}{c|}{$\begin{array}{c}\text { Navettage urbain } \\
\text { vers Saint-Félicien }\end{array}$} \\
\cline { 2 - 13 } & $\begin{array}{c}\text { Taux } \\
2006\end{array}$ & $\begin{array}{c}\text { Diff. 96- } \\
06\end{array}$ & $\begin{array}{c}\text { Taux } \\
2006\end{array}$ & $\begin{array}{c}\text { Diff. 96- } \\
06\end{array}$ & $\begin{array}{c}\text { Taux } \\
2006\end{array}$ & $\begin{array}{c}\text { Diff. 96- } \\
06\end{array}$ & $\begin{array}{c}\text { Taux } \\
2006\end{array}$ & $\begin{array}{c}\text { Diff. 96- } \\
06\end{array}$ & $\begin{array}{c}\text { Taux } \\
2006\end{array}$ & $\begin{array}{c}\text { Diff. 96- } \\
06\end{array}$ \\
\hline Saguenay & & & 1,56 & 0,05 & 0,08 & 0,01 & 0,07 & 0,01 & 0,08 & $-0,08$ \\
\hline Alma & 7,71 & 1,91 & & & 0,32 & $-0,2$ & 0,56 & 0,2 & 0,08 & $-0,49$ \\
\hline Dolbeau-M. & 0,38 & $-0,48$ & 0,94 & 0,51 & & & 1,51 & 0 & 3,4 & 0,27 \\
\hline Roberval & 0 & 0 & 0,69 & $-0,12$ & 0,81 & 0,8 & & & 6,46 & 2,15 \\
\hline Saint-Félicien & 0,91 & $-0,07$ & 0,23 & $-0,05$ & 4,87 & 1,23 & 8,83 & 0,57 & & \\
\hline
\end{tabular}

Quatrièmement, il est surprenant de constater qu'au-delà du navettage, $85,85 \%$ de la population active occupée travaille dans l'une ou l'autre des cinq villes de la région administrative, dont 58,49\% à Saguenay seulement (voir le tableau 4). Ainsi, la quasi-totalité des populations urbaines $(95,65 \%)$ travaillent dans leur ville ou dans une autre ville, alors que près de la moitié des populations rurales occupent un emploi en milieu urbain $(47,61 \%)$. Cette forte concentration urbaine des emplois explique dans une large part la configuration des flux de navettage au Saguenay-Lac-Saint-Jean. Cette réalité laisse également sous-entendre l'existence de vastes bassins d'emplois et la disparition progressive de la distinction entre les milieux urbains et les milieux ruraux, ces derniers devenant progressivement des «quartiers résidentiels » de la ville la plus proche du navettage et de changements d'ordre socioculturel.

Tableau 4 - Travailleurs urbains en 1996 et 2006

\begin{tabular}{|l|c|c|c|c|c|c|}
\hline Population active occupée & $\begin{array}{c}\text { \% se } \\
\text { déplaçant } \\
\text { vers 5 villes } \\
\mathbf{2 0 0 6}\end{array}$ & $\begin{array}{c}\text { \% se } \\
\text { déplaçant } \\
\text { vers 5 villes } \\
\mathbf{1 9 9 6}\end{array}$ & $\begin{array}{c}\text { Différence de } \\
\text { \% 1996-2006 }\end{array}$ & $\begin{array}{c}\text { \% travaillant } \\
\text { dans 5 villes } \\
\mathbf{2 0 0 6}\end{array}$ & $\begin{array}{c}\text { \% travaillant } \\
\text { dans 5 villes } \\
\mathbf{1 9 9 6}\end{array}$ & $\begin{array}{c}\text { Différence de } \\
\mathbf{1 9 9 6 - 2 0 0 6}\end{array}$ \\
\hline Région du Saguenay & 8,16 & 6,64 & 1,52 & 94,27 & 95,43 & $-1,15$ \\
\hline Région du Lac St-Jean & 20,58 & 17,11 & 3,47 & 72,36 & 73,81 & $-1,45$ \\
\hline Zones rurales & 47,61 & 42,80 & 4,81 & 47,61 & 42,80 & 4,81 \\
\hline Zones urbaines & 4,05 & 3,57 & 0,48 & 95,65 & 97,02 & $-1,38$ \\
\hline TOTAL pour le SLSJ & 12,94 & 10,51 & 2,43 & 85,85 & 87,44 & $-1,59$ \\
\hline
\end{tabular}




\section{UNE RURALITÉ PLURIELLE À CATÉGORISER}

À cette étape-ci, nous avons essayé de saisir les flux de navettage et leur évolution à partir de leur mise en corrélations avec des variables comme la distance de la municipalité par rapport à Saguenay ${ }^{18}$, le taux de chômage de même que la population active travaillant dans le domaine tertiaire $^{19}$ (voir le tableau 5). Le coefficient de corrélation de Pearson révèle une relation inverse forte $(-0,71)$ entre le pourcentage de la population active occupée navettant vers Saguenay en 2006 et la distance par rapport à Saguenay et une relation faible entre le pourcentage de la population active occupée se déplaçant quotidiennement vers Saguenay en 2006 et le pourcentage de la population active travaillant dans le secteur tertiaire la même année $(0,25)$. Néanmoins, il n'y a pas de lien significatif entre la croissance de la population travaillant dans le secteur tertiaire entre 1996 et 2006 et la croissance du navettage pendant la même période $(0,09)$. Étrangement, il n'y a pas non plus de relation significative entre le navettage vers Saguenay et le taux de chômage $(-0,06)$. Les municipalités périphériques sont davantage affectées par la crise dans l'industrie forestière et le chômage, mais il semble que cela ne conduit guère à trouver un emploi dans la métropole régionale et à navetter.

Par la suite, nous avons interpellé l'indice de dévitalisation ou de développement des municipalités du Québec mis au point par le MAMROT. Cet indice composite qui s'apparente au célèbre indice de développement humain de l'ONU met en balance les variables suivantes : le taux d'évolution de la population de 2001 à 2006 : le taux de chômage, le taux d'emploi de la population de 15 ans et plus, le pourcentage du revenu provenant de paiement de transfert gouvernemental, la proportion de la population des ménages à faible revenu, le revenu moyen des ménages ainsi que le pourcentage de la population de 15 ans et plus n'ayant pas de diplôme d'études secondaires. À l'échelle de la région administrative, la distance à Saguenay a un effet sur l'indice de développement des municipalités locales $(-0,42$ - corrélation inverse modérée). Dans la même veine, il appert que la présence de navetteurs vers Saguenay présente un certain lien avec l'indice de développement $(0,27$ - corrélation faible) .

Tableau 5 - Corrélations entre le navettage et certaines variables (1996-2006)

\begin{tabular}{|l|c|}
\hline \multicolumn{2}{|l|}{ Coefficient de corrélation - Saguenay } \\
\hline A versus B & $-0,71$ \\
\hline A versus C & $-0,07$ \\
\hline A versus D & 0,25 \\
\hline A: \% de la pop. active occupée navettant vers Saguenay en 2006 \\
\hline B: Distance de la localité par rapport à Saguenay en Km \\
\hline C: Taux de chômage déssaisonalisé (Statistique Canada, 2006) \\
\hline D: \% de la population active travaillant dans le secteur tertiaire en 2006 \\
\hline Coefficient de corrélation - 5 villes du SLSJ combinées \\
\hline A versus B & $-0,24$ \\
\hline A versus C & 0,29 \\
\hline A versus D & 0,45 \\
\hline E versus F & 0,81 \\
\hline D versus B & $-0,48$ \\
\hline D versus E & 0,56 \\
\hline D versus F & 0,6 \\
\hline A: $\%$ de la population active occupée navettant vers le centre urbain le plus proche en 2006 \\
\hline B: Distance de la localité par rapport au centre urbain le plus proche en Km \\
\hline C: \% de la population active travaillant dans le secteur tertiaire en 2006 \\
\hline D: Indice de développement du MAMROT (2008) \\
\hline E: Nouvelles unités résidentielles principales entre 2004 et 2006 \\
\hline F: Population totale en 2006 \\
\hline
\end{tabular}


La vérification de ce modèle a été réalisée à partir d'une analyse à l'échelle de l'ensemble de la région administrative du Saguenay-Lac-SaintJean. Une classification hiérarchique ascendante a été développée afin de procéder à une typologie des 45 municipalités rurales du territoire régional (voir la carte 3 ). Il en résulte quatre classes issues de sept variables. L'interprétation de ce regroupement est effectuée en analysant les moyennes des indicateurs pour chaque groupe (voir le tableau 6). Les variables les plus discriminantes dans cette classification semblent être le nombre d'habitants, l'indice de développement du MAMROT et le nombre de nouvelles résidences principales entre 2004 et 2006. Les effets de la distance au centre urbain le plus proche et la proportion de travailleurs issus du tertiaire sont également non-négligeables. Outre les pôles périurbains qui constituent des quasi-villes, voire des pôles secondaires d'agglomérations urbaines, on remarque trois groupes de villes que l'on peut décrire par les appellations suivantes : espaces suburbains et de villégiature, territoires agro-forestiers en voie de requalification et milieux ruraux dévitalisés.

\section{Carte 3 - Typologie des espaces ruraux}

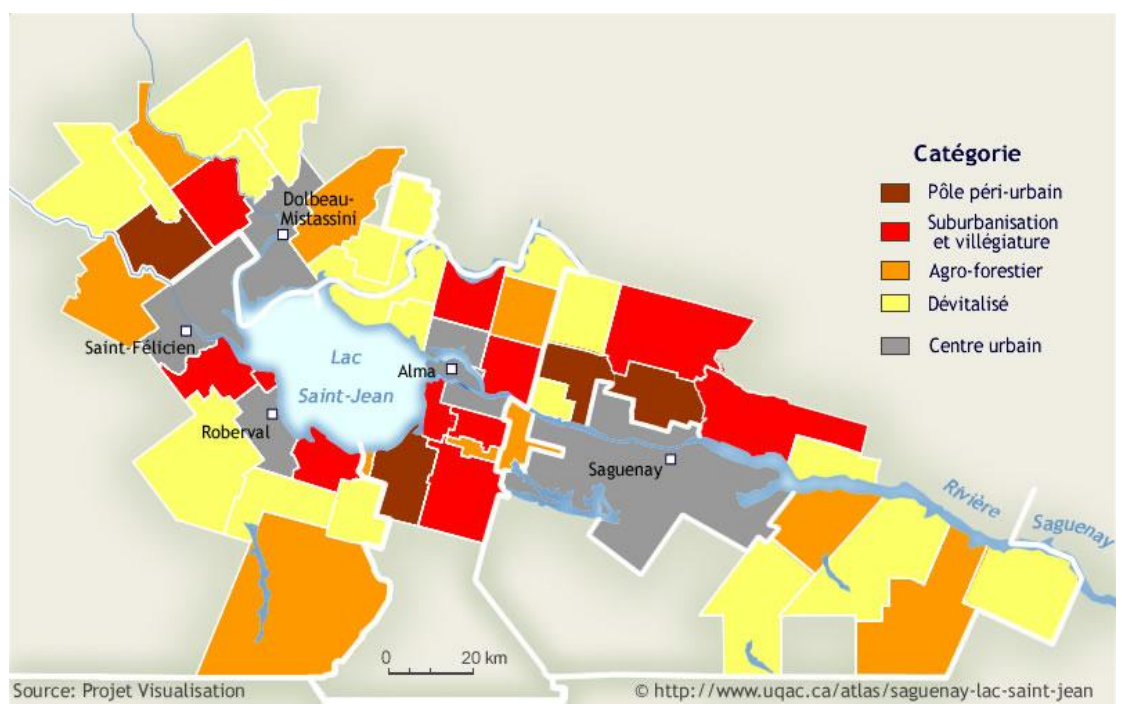

Tableau 6 - Valeurs moyennes des variables selon les groupes identifiés

\begin{tabular}{|l|c|c|c|c|}
\hline $\begin{array}{l}\text { Groupes / } \\
\text { Indicateurs }\end{array}$ & $\begin{array}{c}\text { Groupe 1 - } \\
\text { Rural dévitalisé }\end{array}$ & $\begin{array}{c}\text { Groupe 2 - } \\
\text { Agro-forestier en } \\
\text { requalification }\end{array}$ & $\begin{array}{c}\text { Groupe 3 - } \\
\text { Suburbanisation } \\
\text { et villégiature }\end{array}$ & $\begin{array}{c}\text { Groupe 4 - } \\
\text { Pôle périurbain }\end{array}$ \\
\hline $\begin{array}{l}\text { Population totale } \\
\text { (2006) }\end{array}$ & 1198,4 hab. & 603,4 hab. & 2141,1 hab. & 3878,75 hab. \\
\hline $\begin{array}{l}\% \text { de travailleur dans le } \\
\text { secteur tertiaire (2006) }\end{array}$ & $61,0 \%$ & $54,8 \%$ & $64.9 \%$ & $65,2 \%$ \\
\hline $\begin{array}{l}\text { Indice de développement } \\
\text { du MAMROT (2006) }\end{array}$ & $-1,52$ & $-4,27$ & 1,31 & 0,45 \\
\hline $\begin{array}{l}\% \text { de navettage } \\
\text { (5 villes) }\end{array}$ & $46 \%$ & $38 \%$ & $51 \%$ & $45 \%$ \\
\hline $\begin{array}{l}\text { Nouvelles résidence } \\
\text { principale (04-06) }\end{array}$ & 7,5 & 3,8 & 24,9 & 38,0 \\
\hline $\begin{array}{l}\text { Nouvelles résidence } \\
\text { secondaire (04-06) }\end{array}$ & 9,4 & 5,6 & 13,5 & 1,8 \\
\hline $\begin{array}{l}\text { Distance au centre urbain } \\
\text { le plus proche }\end{array}$ & $31,2 \mathrm{~km}$ & $36,8 \mathrm{~km}$ & $16,7 \mathrm{~km}$ & $23,9 \mathrm{~km}$ \\
\hline
\end{tabular}




\section{CONCLUSION}

L'étude du navettage dans la région administrative québécoise du Saguenay-LacSaint-Jean démontre qu'une part croissante de la population active est mobile et souvent sur de grandes distances.

En ce qui concerne les résultats, l'étude du navettage dans la région administrative québécoise du Saguenay-Lac-Saint-Jean démontre qu'une part croissante de la population active est mobile et souvent sur de grandes distances. En second lieu, la forte concentration des emplois en milieu urbain et l'attractivité croissante des pôles urbains ont été mises en relief, et ce, même pour des villes petites et moyennes en milieu périphérique comme celles de la région étudiée. À cet égard, la forte primauté de Saguenay dans le système territorial régional a été confirmée. D'ailleurs, l'éloignement par rapport à cette ville explique largement les variations dans l'indice de développement des collectivités locales de type rural. Nos recherches rejoignent les travaux sur ce thème, à plusieurs égards.

Troisièmement, nous avons constaté que l'influence urbaine à l'ère de la métropolisation induit une catégorisation des milieux ruraux selon trois classes: espaces suburbains et de villégiature, territoires agro-forestiers en voie de requalification ou milieux ruraux dévitalisés. Des auteurs ont déjà démontré la diversité des espaces ruraux. Toutefois, les effets de la présence des villes y étaient plutôt négligés. Par opposition, les travaux sur les villes et l'espace rural se limitent à la distance à la ville. Notre catégorisation de l'espace rural en trois types a été testée par l'intermédiaire d'analyses factorielles multivariées, à l'échelle du SaguenayLac-Saint-Jean. Le navettage ressort comme l'un des facteurs clefs de la structuration des espaces ruraux et de l'espace régional dans son ensemble.

La question qui se pose maintenant est la suivante : est-ce que la polarisation de Saguenay et ses effets sur la qualité des milieux ruraux est reliée à la métropolisation ou est-ce simplement une forme traditionnelle d'influence urbaine? Au-delà de la fragmentation sociospatiale intramétropolitaine attribuée à ce processus, la métropolisation implique une tertiarisation de l'économie. Au Saguenay-Lac-Saint-Jean, cette tertiarisation se fait en partie par la crise forestière depuis une dizaine d'années. Le partage des revenus provenant des emplois tertiaires principalement concentrés dans les villes devient alors un important moyen de répartition de la richesse à travers l'économie résidentielle. Le navettage apparaît de la sorte comme le mécanisme régulateur et le révélateur de cette distribution géographique des revenus d'emplois tertiaires. L'achat de résidences principales ou secondaires, la consommation locale ainsi que le récréotourisme de courte durée remplacent progressivement les emplois industriels dans les municipalités rurales, notamment les emplois liés à l'industrie forestière dans l'exemple du Saguenay-LacSaint-Jean. On peut déplorer, voire dénoncer, cette domination des villes dans les rapports urbain-rural, mais celle-ci semble être une réalité inéluctable qui consacre une forme de régionalisation des dynamiques socioéconomiques.

Les territoires ne s'organiseraient plus en deux mondes façonnés par la densité de population, les profils socioprofessionnels et certains traits culturels, mais davantage en plusieurs types fondés sur la distance au pôle d'emploi principal, le niveau

d'intégration à l'économie mondialisée de même que les attributs du paysage.

$\mathrm{Au}$ fond, il s'agit d'un débat anachronique dans la mesure où la dualité urbain-rural n'existe plus vraiment. L'économie de marché postindustrielle, mise en exergue par la métropolisation, structure les territoires de manière différenciée, mais selon une méthode standardisée. Les territoires ne s'organiseraient plus en deux mondes façonnés par la densité de population, les profils socioprofessionnels et certains traits culturels, mais davantage en plusieurs types 
fondés sur la distance au pôle d'emploi principal, le niveau d'intégration à l'économie mondialisée de même que les attributs du paysage. On devrait donc parler dorénavant de degrés d'urbanité ou de ruralité et non d'états urbain ou rural pour les territoires locaux. Quoi qu'il en soit, l'avènement d'une société urbaine affecte maintenant l'ensemble des milieux, selon une intensité variable. Seuls les grands ensembles naturels peu habités et les petites enclaves socioculturelles très isolées sont imperméables à ces dynamiques de recompositions territoriales. Toutefois, la terminologie géographique et les représentations des populations tardent à s'adapter à cette réalité.

\section{BIBLIOGRAPHIE ET NOTES}

${ }^{1}$ Martin Simard est géographe et urbaniste. Il travaille comme professeur titulaire à l'Université du Québec à Chicoutimi en plus d'être membre régulier du Centre de recherche sur le développement territorial.

${ }^{2}$ Yves Chiricota est mathématicien et informaticien. Il œuvre comme professeur titulaire à l'Université du Québec à Chicoutimi.

${ }^{3}$ Carl Brisson est géographe. Il agit comme professionnel de recherche au Laboratoire d'expertise et de recherche en géographie appliquée de l'Université du Québec à Chicoutimi.

${ }^{4}$ François Queyroi est informaticien. Il est rattaché au Laboratoire bordelais de recherche en informatique Université de Bordeaux I et II.

${ }^{5}$ Charron, M. (2006). La complexité des phénomènes spatiaux, Cahiers de géographie du Québec, vol. 50, no. 141,2006 , p. 327-335.

${ }^{6}$ Ali, K., Olfert, M.R. et Partridge, M.D. (2007). Urban Footprints in Rural Canada : Employment Spillovers by City Size, Regional Studies, 1360-0591, published on 21 January 2010, $32 \mathrm{p}$.

${ }^{7}$ Hall, H. M. et Hall, P. V. (2008). Decline and no growth: Canada's forgotten urban interior, Canadian Journal of Regional Science, vol. 31, no. 1, p. 102-116.

${ }^{8}$ Voir Berubé, A., Katz, B., Lang, R., 2003, Redefining Urban and Suburban America.

${ }^{9}$ Édouard, J.-C. et Mainet, H. (2007). L'Allier face à la métropolisation, Cahiers de géographie du Québec, vol. 51, no. 143, p. 177-195.

${ }^{10}$ Elissalde, B. (2004), «Métropolisation », Hypergéo [En ligne], http://www.hypergeo.eu/ spip.php?article75, consulté le 10 août 2011.

${ }^{11}$ Fusco, G. et Scarella, F. (2009). Mobilités potentielles et émergence de structures réticulaires en région Provence - Alpes - Côte d'Azur, L'Espace géographique, vol. 38, no. 3, p. 297-282.
${ }^{12}$ Sénécal, G. (2007) «Métaphores et modèles en géographie urbaine: le continuum de l'école de Chicago à celle de Los Angeles ", Annales de géographie, no. 657, p. 513-532.

${ }^{13}$ Partridge, M. D., Ali, K. et Olfert, M. R. (2010). Rural-to-Urban Commuting: Three Degrees of Integration, Growth and Change, vol. 41

${ }^{14}$ Hamel, P. (2010). «Les métropoles et la nouvelle critique urbaine », Métropoles [En ligne], URL: http://metropoles.revues.org/4317, consulté le 16 juillet 2010, p. 3.

${ }^{15}$ Gauthier, M.-J., Brisson, C., Dion, M., Roch, A. et Chamberland, C. (2000). La place des centres urbains dans l'organisation de l'espace régional $d u$ Saguenay-Lac-St-Jean, Rapport de recherche, Université du Québec à Chicoutimi, 30 pages.

${ }^{16}$ MAMROT (2008). Pour une plus grande prospérité et vitalité de nos municipalités. Plan d'action gouvernemental à l'intention des municipalités dévitalisées, Ministère des Affaires municipales, des régions et de l'occupation du territoire, Québec, $60 \mathrm{p}$.

${ }^{17}$ Pour les fins de cette étude, les espaces ruraux se définissent comme les municipalités de moins de 10000 habitants, soit toutes les municipalités locales à l'exception des villes de Saguenay, Alma, DolbeauMistassini, Roberval et Saint-Félicien en ce qui concerne le Saguenay-Lac-St-Jean. Par voie de conséquence, ces cinq municipalités constituent les espaces considérés comme étant urbains.

${ }^{18}$ Pour calculer la distance entre les municipalités locales, nous avons utilisé un point central, ou centroïde, pour chacun des territoires municipaux, point obtenu à l'aide d'outils informatiques.

${ }^{19}$ Tous les coefficients de corrélation ont été réalisés à partir de séries statistiques qui excluent la Ville de Saguenay. 


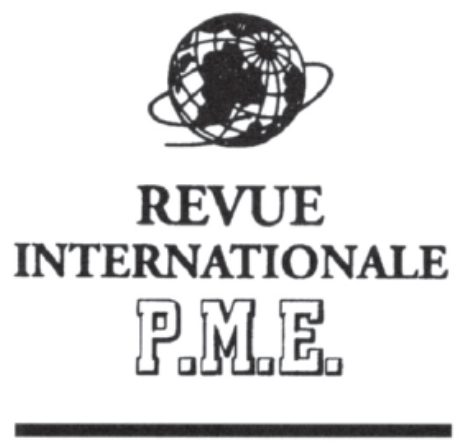

Volume 24, numéro 1, 2011

\title{
SOMMAIRE
}

\author{
ÉDITORIAL \\ Louis Raymond
}
Perceptions culturelles et intention d'entreprendre : une comparaison entre des étudiants brésiliens et français
Saulo D. Barbosa, Walter Marinho de Oliveira, Alain Fayolle et Francisco Vidal Barbosa EMLYON, CERAG Univesité Pierre-Mendès-France et CEPEAD Universidade Federal de Minas Gerais

Autonomie des salariés, participation aux décisions et sentiment d'insécurité : une analyse de la petite entreprise dans 25 pays européens

Yvan Barel, Emmanuel Dion et Sandrine Fremeaux

Université de Nantes et Audencia École de management

\author{
Repenser l'échec entrepreneurial des petites entreprises émergentes : \\ proposition d'une typologie s'appuyant sur une approche intégrative \\ Ali Smida et Nabil Khelil \\ Université Paris XIII et IAE de Caen \\ Valoriser le potentiel humain ou délocaliser : \\ quel choix pour les PME? \\ Virginie Gallego et Lynda Saoudi \\ CERAG Université Pierre-Mendès-France et ESC Clermont-Ferrand \\ La gestion stratégique des ressources humaines \\ dans les PME françaises : quelle place et quelle évolution? \\ Abdelwahab Ait Razouk et Mohamed Bayad \\ ESC Bretagne Brest et IAE de Nancy \\ COMPTES RENDUS \\ INFORMATIONS
}

TABLES DES MATIÈRES DES REVUES ASSOCIÉES 